\title{
Partnerships for Success: An Evaluation of Head to Toe, a Family Centered Childhood Obesity Intervention that Partners with an Obesity Clinic
}

\author{
Melody Schaeffer, Claire Cioni, Jill Schnurbusch, Nicole Kozma, Sarah Reinhold and Nila Garba \\ St. Louis Children's Hospital, Child Health Advocacy and Outreach
}

\begin{abstract}
The prevalence of childhood obesity is a growing epidemic in the United States and as of 2014 its prevalence among children ages two to 19 was $17.0 \%$. Without intervention, obese children are at risk for negative health consequences and for the increased likelihood of staying obese through adulthood. St. Louis Children's Hospital provides HTT (Head to Toe), an interactive 12-session fitness, nutrition, and social well-being class focuses on adopting healthier lifestyles through daily exercise, and proper nutrition. One program referral method is through a partnership with the hospital's HSC (Healthy Start Clinic), a multi-disciplinary obesity clinic. Pretest and posttest assessments are used to measure changes in body metrics, health behaviors, knowledge, readiness to change, and self-concept. Between August 2011 and May 2016, 485 children and at least one parent or guardian with them were enrolled in the program, and up to 235 children completed at least one pretest- or post-assessment. Analysis determined multiple statistically significant improvements in body metrics, health behaviors, knowledge, including questions related to nutrition and food guidelines, readiness to change assessment, and self-concept. Total knowledge increased by $22 \%$; questions related to nutrition increased by $28.2 \%$, social emotional by $18.9 \%$ and physical activity by $17.8 \%$. Findings suggest that the Head to Toe program is effective in improving nutrition, physical activity, social emotional knowledge and behaviors among participants. Childhood obesity is a growing epidemic and creating a relationship with a pediatric obesity clinic can increase the total number of children and teens that are introduced to weight management programs.
\end{abstract}

Key words: Childhood obesity, exercise, nutrition, weight, wellness, mental health.

\section{Introduction}

The prevalence of childhood obesity is a growing epidemic in the United States. Between 2011 and 2014, the obesity prevalence among children ages two to 19 was $17.0 \%$, which is up from $13.9 \%$ between 1999 and 2000 [1]. Healthy people 2020 has set a goal to reduce this proportion among children ages two to 19 to $14.5 \%$ or less by year 2020 [2]. Obesity is defined as BMI (body mass index) $\left(\mathrm{kg} / \mathrm{m}^{2}\right)$ being greater than or equal to the 95th percentile range relative to other children of the same gender and age [3].

Children above the 75th percentile for BMI at 54

Corresponding author: Melody Schaeffer, MPH, supervisor of community benefit and evaluation, research fields: weight management, injury, and birth outcomes. months are at an increased risk of being overweight (85th to 95th percentile) by age 12 [4]. Overweight or obese older children are then more likely to become obese adults than non-overweight or obese children [5, 6]. Obesity prevalence also increases at each developmental stage. And $8.9 \%$ of children ages two to five are obese, and that proportion increases to $17.5 \%$ for children ages six to 11 . Prevalence among those aged 12 to 19 is even higher at 20.5\% [1]. This indicates a need for intervention at an early age to prevent the increase in prevalence of obesity at adolescence and into adulthood and to prevent the adverse health consequences of obesity. Comorbidities of childhood obesity include: cardiovascular disease risk factors such as high blood pressure and high cholesterol [6, 7], pre-diabetes and type 2 diabetes [6, 8], sleep apnea, orthopedic stress 
on the developing skeleton [7] and psychosocial distress such as depression and lower health related quality of life [7, 9]. Weight management interventions can improve present quality of life for the child and prevent future health complications.

Research indicates that the most effective pediatric weight management programs involve both parents and children [10] and are multidisciplinary in nature, combining nutrition, physical activity and behavioral modification [11]. Parents have a great deal of influence over the family's lifestyle, and when parents and children manage their weight at the same time, parents act as models for healthy eating and activity behaviors; this in turn underpins the child's likelihood of adherence to positive lifestyle changes [12]. Data show that including parents in the intervention/program improves child weight loss, as compared with an intervention/program with the child alone [12].

Results are mixed for weight management programs that focus on BMI and weight reduction as the primary outcomes, and if effective, results are usually not sustained past 12 to 18 months post-intervention [13]. Focusing on small, sustainable lifestyle changes may have a greater long term effect on obesity than programs that focus on weight loss and BMI as primary outcomes alone [11]. Participants of weight management programs need to be equipped with skills, abilities, and knowledge to make continual progress toward healthier lifestyles.

Another important aspect for successful pediatric weight management is to understand where potential participants are on the readiness to change spectrum. Interventions can then be targeted to those most likely to succeed. According to the TTM (trans-theoretical model), there are five stages of change that people pass through when making a lifestyle change: pre-contemplation, contemplation, preparation, action, and maintenance. Those in the pre-contemplation or contemplation stage are less likely to successfully instigate a long-lasting change than someone in the preparation, action, or maintenance stage [14]. Past studies utilizing TTM for weight management have reported positive results for reduction of fat intake, increases in fruit and vegetable intake, and increase in physical activity [15].

In St. Louis, Missouri, St. Louis Children's Hospital provides a multidisciplinary obesity clinic, called the HSC (Healthy Start Clinic). St. Louis Children's Hospital nationally serves the health care needs of children, from infancy to adolescence, advocates on behalf of children and families, and serves patients from all 50 states and more than 80 countries. The HSC includes a staff of physician, psychologist, dietitian, registered nurse, social worker, and a representative of the childhood weight management program called HTT (Head to Toe). The relationship between the HSC and HTT began in 2015 and if the patient meets the requirements for the intervention, such as age, distance from St. Louis Children's Hospital, and readiness to change, the HTT representative gives an introduction to the program detailing the logistics and expectations of participating. As of 2017, 54\% off HSC patients meet the requirements and are given the HTT introduction.

The purpose of HTT is to provide a family-based weight management program that increases both behavior and knowledge of healthier lifestyle choices which address risk factors for chronic diseases like hypertension and diabetes. HTT was created in 1996 with two families, a dietitian, and a physical therapist. The program has grown to include paid staff, a set curriculum, and replication partners across the state of Missouri. Program curriculum for HTT was developed in 1996 by hospital staff based on literature reviews. The curriculum is delivered orally by a cross-trained team of hospital staff including a registered dietitian, an exercise specialist, and a licensed social worker. Through twelve, 60 minute sessions over the course of 16 weeks, a team of trained professionals teach participants how to manage their weight and encourage them to have a positive self-image as their 

Obesity Intervention that Partners with an Obesity Clinic

bodies grow. Participants set goals to increase regular exercise, practice healthy eating, and increase self-esteem. The curriculum was developed using CDC (Centers for Disease Control and Prevention) guidelines for physical activity and USDA (United States Department of Agriculture) Center for Nutrition Policy and Promotion guidelines for nutrition. In 2013, the program was deemed "effective practice" by the Healthy Communities Institute, and in 2016, the program was upgraded to "evidence based."

Participants are recruited by an area physician referral, the HSC and word of mouth based on the following ideal criteria: BMI above the 85th percentile (classified as overweight or obese), in the preparation or action phase on the readiness to change assessment, and residing within a 50 mile radius of the St. Louis Children's Hospital, but any interested family is welcome to join the program. The program hosts a kid class (ages 8 to 12) and a teen class (ages 13 to 17), and there are a maximum of 15 child participants per class. At least one parent or guardian attends each class with the child participant, and siblings are also welcome to attend. This reinforces the lifestyle component of the program by involving the entire family in health behavior changes. Classes each week are focused on a different topic-nutrition, physical activity, or social work-and are led by the appropriate trained professional on the team, which consists of a registered dietitian, an exercise specialist, and a licensed social worker. The trained professionals attend all classes in order to create a familiar and supportive environment from week to week.

The first eight of the twelve classes meet weekly, and the final four meet every other week. Incorporating a tapering phase into interventions prevents relapse of bad habits by providing support and by reviewing previous lessons; this has been shown to help individuals maintain changes that occurred in the intervention [16]. The program costs $\$ 60$, and families are fully reimbursed if they achieve perfect attendance.
The purpose of this study is to evaluate the effect HTT has on creating sustainable changes in lifestyles among program participants. Additionally, this study will investigate the relationship between the HSC and HTT. No previous study has yet to provide estimates on the benefit HTT has on children and families. We hypothesize that participants will create positive lifestyle changes after having completed the program.

\section{Materials and Methods}

In 2015, St. Louis Children's Hospital's HSC included an introduction to the program as part of patients' initial visits to the clinic and $15.7 \%$ of participants have been referred from 2015 to 2016 through the clinic.

Once enrolled into HTT, staff capture changes in body metrics, knowledge, health behaviors, readiness to change, and self-concept, pretest and posttest assessments. Survey question development was based on validated literature reviews, was externally evaluated to determine appropriate grade level language usage and included multiple choice and ranking questions.

The body metrics assessment evaluates the child's height, weight, and BMI, as well as physical abilities such as hamstring flexibility, number of sit ups in 30 seconds, number of push-ups in 30 seconds, and a 30 second running $\mathrm{VO}_{2}$ max exercise. The knowledge assessment is a 13 questions true-false test containing fitness, nutrition, and social emotional items. Behavior change is assessed with an online 28 questions test that asks about current dietary behaviors, fitness routines, media usage and family time. In the readiness to change assessment, Prochaska's TTM is used to identify participants' readiness to adopt healthy behaviors both before and after the program. Items assess current levels of: exercise, eating fruits and vegetables, family support, and how future activities will affect the families' ability to maintain a healthy lifestyle. Items for all assessments were developed based on literature reviews for each topic. 
Self-concept is assessed by the Piers Harris 2.0 to provide an overall view of an individual's self-perception and to help identify participants who may require further testing and possible treatment. The six categories assessed on Piers Harris are: behavioral adjustment, intellectual and school status, physical appearance and attributes, freedom from anxiety, popularity, and happiness and satisfaction. Knowing where participants are on the spectrum of self-concept equips the program leaders with an understanding of how best to reach the participants.

All of the assessments take 30 minutes to complete and every child in the program, ages 8 to 18 , received both the pretest and the posttest. The pretest assessments were administered during the first session of HTT and the posttest assessments were administered at the last session, both by paper and pencil.

Completed health behavior and readiness to change surveys are entered into the Qualtrics Insight Platform for data management and body metrics, knowledge, and self-concept assessments are entered into excel. For this study, a data file from 2011-2016 was exported from Qualtrics and imported into SPSS 22.0 for data cleaning and analysis. Statistical analysis was conducted to determine the mean test scores for knowledge change from pretest and post test data.

Results from pre- and post-assessments were compared using paired $t$-tests, independent samples $t$-tests, and chi-square analysis for categorical items on the assessments. Statistically significant results are identified if the $p$-value is less than 0.05 . Items on the assessments were adapted over the five years of program implementation, causing differences in samples sizes on particular items. No surveys were excluded from the analysis.

\section{Results and Discussion}

Between August 2011 and May 2016, 485 children and at least one parent or guardian were enrolled in the program, and up to 235 children completed at least one pre- or post-assessment. Along with item adaptation, absences year to year for pre- and post-assessments, lack of follow-up, and refusal to answer some questions resulted in different samples sizes for assessment items. For this reason, paired $t$-tests were not always possible and independent samples $t$-tests were used in those instances.

Participants improved from pre- to post-test on all body metric assessments, with statistically significant improvements for number of push-ups in 30 seconds, $\mathrm{VO}_{2}$ max, and sit and reach (Table 1). Average BMI and average weight (pounds) decreased from pre- to post-test (31.95 to 31.33 and 169.37 to 169.31, respectively), but these differences were not statistically significant. Body metric results were analyzed with a paired samples $t$-test.

Participants also showed improvements in health behaviors (Table 2). For nutrition related behaviors, there were statistically significant improvements for: number of days consuming fruits and vegetables, number of days eating as a family, cups of water consumed per day, and number of days per week planning and prepping meals. For social emotional health behaviors, participants saw improvements in the positive direction on three of four items, and

Table 1 Change in mean results, N, 95\% CIs, and $p$ values for body metrics assessments.

\begin{tabular}{lllll}
\hline Metric & Mean difference & $\mathrm{N}$ & $95 \%$ CI & $p$ \\
\hline Push ups in 30 seconds & +1.04 & 155 & -1.57 to -0.50 & $0.000^{*}$ \\
$\mathrm{VO}_{2}$ max "beep test" & +1.70 & 93 & -2.90 to -0.49 & $0.006^{*}$ \\
Sit and reach (centimeters) & +1.80 & 159 & -2.57 to -1.04 & $0.000^{*}$ \\
Sit ups in 30 seconds & +0.66 & 157 & -1.39 to 0.076 & 0.079 \\
Weight (pounds) & -0.06 & 137 & -6.07 to 6.19 & 0.985 \\
BMI & -0.62 & 156 & -0.38 to 1.62 & 0.223 \\
\hline
\end{tabular}

*statistical significance $(p<0.05)$. 

Obesity Intervention that Partners with an Obesity Clinic

Table 2 Change in mean results, N, 95\% CI, and $p$ values for health behaviors assessments.

\begin{tabular}{lllll}
\hline Health behavior & Mean difference & $\begin{array}{l}\text { N } \\
\text { pre-, post- }\end{array}$ & 95\% CI & $p$ \\
\hline Nutrition & & & & \\
\hline Days/week eating at least 5 fruits and vegetables & +1.04 & 140,28 & -1.87 to -0.22 & $0.014^{*}$ \\
Fruits and vegetables eaten on average day & +0.50 & 92,64 & -1.42 to 0.43 & 0.290 \\
Days/week eating as a family & +1.88 & 229,90 & -3.02 to -0.73 & $0.001^{*}$ \\
Cups of water per day & +3.22 & 235,92 & -4.79 to -1.65 & $0.000^{*}$ \\
Sugar sweetened beverages per day & -0.66 & 91,64 & -0.80 to 2.13 & 0.371 \\
Cups of soda per day & -0.56 & 134,27 & 0.19 to 0.93 & $0.004^{*}$ \\
Cups of Gatorade per day & -0.19 & 134,27 & -0.31 to 0.70 & 0.453 \\
Cups of juice per day & -0.30 & 139,26 & -0.55 to 1.15 & 0.488 \\
Times per week eating fast food & +0.33 & 232,91 & -0.97 to 0.31 & 0.315 \\
Days/week planning and prepping food & +1.36 & 227,88 & -2.46 to -0.27 & $0.015^{*}$ \\
Meals eaten last week & -0.58 & 92,61 & -1.47 to 2.63 & 0.577 \\
Days/week eating within two hours of waking & +0.10 & 90,64 & -1.55 to 1.34 & 0.890 \\
\hline Social work & & & & -2.53 to 0.065 \\
\hline Days/week 10 minutes conversations with family & +1.23 & 235,92 & 0.062 \\
Days/week positive self-talk & +2.46 & 233,92 & -3.73 to -1.20 & $0.000^{*}$ \\
Days/week talking about media messages & +0.60 & 231,92 & -1.60 to 0.40 & 0.238 \\
Days/week feeling stressed & +1.42 & 227,87 & -2.81 to -0.02 & $0.047^{*}$ \\
\hline Physical activity & & & & -1.13 to -0.15 \\
\hline Days/week physically active & +0.64 & 232,89 & -2.14 to -0.38 & $0.010^{*}$ \\
Days/week walking 10,000 steps & +1.26 & 126,24 & -0.25 to 0.92 & 0.260 \\
Days/week physically active with family & -0.33 & 94,63 & & \\
\hline * & & & & \\
\hline
\end{tabular}

*statistical significance $(p<0.05)$.

improvements were positively statistically significant for number of days per week engaging in positive self-talk. Number of days per week feeling stressed was statistically significant, but in the negative direction. Participants improved physical activity health behaviors in three of the four assessments, and there were statistically significant improvements for the number of days per week they were physically active and number of days per week they walked 10,000 steps. Health behavior results were analyzed with an independent samples $t$-test.

There were three items on the health behavior assessment that had categorical response options and were assessed with a chi-square assessment (Table 3). There was a statistically significant difference from pre- to post-test in how participants responded to the question, "How often do you read nutrition labels on the package of the food you eat?" The responses for the other two items (assessing average time spent exercising on days they were physically active and average time per day on "screens") were not statistically significantly different from pre- to post-test.

On the knowledge assessment, overall, participants showed statistically significant improvements from pre-test (57.5\%) to post-test $(79.6 \%)$ (Table 4). Participants also statistically significantly improved each sub-category of the test: nutrition (mean change M: 28.21\%), social emotional (M: 18.91\%) and physical activity (M: 17.76\%). The largest increase in knowledge was for the question, "True or false, to lose one pound, you must burn 1,000 calories?” with a $60 \%$ increase. There were also statistically significant improvements on 13 of the 18 individual questions on the knowledge test. The knowledge test was assessed with a paired sample $t$-test.

On Proschaka's readiness to change assessment, 
Table 3 Chi Squares analysis, $\mathrm{N}$, and $\boldsymbol{p}$ values for categorical health behaviors.

\begin{tabular}{llll}
\hline Health behavior & $\begin{array}{l}\text { Pearson } \\
\text { chi-square }\end{array}$ & $\begin{array}{l}\mathrm{N} \\
\text { pre-, post- }\end{array}$ & $p$ \\
\hline $\begin{array}{l}\text { How often do you read the nutrition labels on the packages of the food you eat? } \\
\begin{array}{l}\text { On the days that you do take part in physical activity or exercise, how much time } \\
\text { in a day do you normally spend doing these activities? }\end{array}\end{array}$ & $\begin{array}{l}0.166 \\
\begin{array}{l}\text { How much time in a day do you spend in front of the television, computer or } \\
\text { video games? }\end{array}\end{array}$ & $23.027^{*}$ \\
\hline
\end{tabular}

*statistical significance $(p<0.05)$.

Table 4 Change in mean results, N, 95\% CI, and $p$ values for knowledge assessments.

\begin{tabular}{|c|c|c|c|c|}
\hline Knowledge item & $\begin{array}{l}\text { Mean difference } \\
\text { (\%) }\end{array}$ & $\mathrm{N}$ & $95 \%$ CI & $p$ \\
\hline \multicolumn{5}{|l|}{ Nutrition } \\
\hline A balanced diet should include lots of plant foods. & +15.45 & 123 & -0.27 to -0.04 & $0.009 *$ \\
\hline Food labels list the amount of calories, fat, protein, and number of servings. & +12.82 & 39 & -0.26 to 0.00 & 0.058 \\
\hline Fruits and vegetables are good for you because they are high in calories. & +35.71 & 84 & -0.47 to -0.24 & $0.000 *$ \\
\hline It takes 5 minutes for your stomach to tell your brain it is full. & +40.65 & 123 & -0.61 to -0.30 & $0.000^{*}$ \\
\hline You only need to drink 4 cups of water every day. & +13.01 & 123 & -0.22 to -0.04 & $0.003^{*}$ \\
\hline Overall nutrition & +28.21 & 123 & -0.33 to -0.23 & 0.000 \\
\hline \multicolumn{5}{|l|}{ Social emotional } \\
\hline S.M.A.R.T. goals should be measurable and attainable. & +26.02 & 123 & -0.35 to -0.17 & $0.000 *$ \\
\hline $\begin{array}{l}\text { Sending an email or text is a positive way to use technology to start a } \\
\text { conversation. }\end{array}$ & +24.40 & 123 & -0.35 to -0.13 & $0.000 *$ \\
\hline $\begin{array}{l}\text { Punching someone, yelling, or breaking something are all positive ways to deal } \\
\text { with stress. }\end{array}$ & +5.70 & 123 & -0.13 to -0.02 & 0.144 \\
\hline $\begin{array}{l}\text { Most decision making is not affected by what I see and hear on TV, in movies, } \\
\text { music, video games or the internet. }\end{array}$ & +19.51 & 123 & -0.30 to -0.09 & $0.000 *$ \\
\hline Overall social emotional & +18.91 & 123 & -0.24 to -0.14 & 0.000 \\
\hline \multicolumn{5}{|l|}{ Physical activity } \\
\hline To lose one pound, you must burn 1,000 calories. & +56.95 & 84 & -0.58 to -0.44 & $0.000 *$ \\
\hline Examples of aerobic exercise are lifting weights, stretching, and sit ups. & +14.75 & 122 & -0.24 to -0.05 & $0.003^{*}$ \\
\hline You should exercise 30-60 minutes per day. & +5.13 & 39 & -0.12 to 0.02 & 0.160 \\
\hline Practicing flexibility can keep your muscles and joints from becoming stiff. & +2.38 & 84 & -0.10 to 0.05 & 0.530 \\
\hline Strength training should be done 4 days a week. & +30.08 & 123 & -0.40 to -0.20 & $0.000^{*}$ \\
\hline It is recommended that you should walk 20,000 steps per day. & +28.21 & 39 & -0.45 to -0.12 & $0.001 *$ \\
\hline Aerobic exercise helps your heart and lungs work better. & 0 & 20 & -0.21 to 0.21 & 1.000 \\
\hline Increasing the amount of muscle on your body makes you burn more calories. & +21.88 & 64 & -0.38 to -0.06 & $0.009 *$ \\
\hline Regular exercise can improve your bones, skin, and mood. & +20.51 & 39 & -0.36 to -0.05 & $0.010^{*}$ \\
\hline Overall physical activity & +17.76 & 123 & -0.23 to -0.13 & 0.000 \\
\hline Overall knowledge & +22.01 & 123 & -0.25 to -0.19 & $0.000^{*}$ \\
\hline
\end{tabular}

*statistical significance $(p<0.05)$.

there was a statistically significant difference in participants' readiness to increase fruit and vegetable consumption and level of how future activities will affect the families' ability to maintain a healthy lifestyle (Table 5). Readiness to change results was assessed with a chi-square analysis.

On the Piers Harris assessment, participants improved their scores on all subsections of the test.
However there were no statistically significant improvements. Piers Harris results (Table 6) were assessed with an independent sample $t$-test. The average inconsistent response index and average response bias index were both within normal ranges on the pre- and post-assessment, indicating that participants did not respond to the Piers Harris items at random or yes-only/no-only. 
Table 5 Chi square analysis, $\mathrm{N}$, and $p$ values for readiness to change assessments.

\begin{tabular}{|c|c|c|c|}
\hline Readiness to change indicator & $\begin{array}{l}\text { Pearson } \\
\text { chi-square }\end{array}$ & $\begin{array}{l}\mathrm{N} \\
\text { pre-, post- }\end{array}$ & $p$ \\
\hline Readiness to engage in physical activity & 7.772 & 187, 96 & 0.105 \\
\hline Readiness to increase fruit and vegetable consumption & 14.797 & 187, 95 & $0.005^{*}$ \\
\hline Current level of support from family & 5.652 & 187, 96 & 0.227 \\
\hline $\begin{array}{l}\text { For the next four months, activities such as work, homework, sports, travel times, } \\
\text { tutoring or other activities will affect my family's ability to achieve or maintain a } \\
\text { healthy lifestyle? }\end{array}$ & 18.904 & 187, 95 & $0.001 *$ \\
\hline
\end{tabular}

*statistical significance $(p<0.05)$.

Table 6 Change in mean results, N, 95\% CI, and $p$ values for piers harris assessment.

\begin{tabular}{lllll}
\hline Self-concept domain & Mean difference & $\begin{array}{l}\mathrm{N} \\
\text { pre-, post- }\end{array}$ & 95\% CI & $p$ \\
\hline Inconsistent responding index & -1.05 & 177,138 & -0.56 to 2.66 & 0.202 \\
Response bias & -0.06 & 177,138 & -1.98 to 2.11 & 0.952 \\
Behavioral adjustment & +5.79 & 177,138 & -14.07 to 2.50 & 0.170 \\
Intellectual and school status & +0.50 & 177,138 & -2.50 to 1.50 & 0.624 \\
Physical appearance and attributes & +2.13 & 177,138 & -4.31 to 0.05 & 0.056 \\
Freedom from anxiety & +1.45 & 177,138 & -3.82 to 0.92 & 0.229 \\
Popularity & +1.87 & 177,138 & -4.23 to 0.48 & 0.120 \\
Happiness and satisfaction & +2.39 & 177,138 & -4.35 to -0.44 & $0.017^{*}$ \\
Total & +1.62 & 177,138 & -4.02 to 0.76 & 0.181 \\
\hline
\end{tabular}

*statistical significance $(p<0.05)$.

\section{Conclusion}

HTT was created to target the growing childhood obesity epidemic in the United States. Without intervention, obese children are at risk for negative health consequences and for the increased likelihood of staying obese through adulthood. This family-based weight management program helps families learn to make healthier lifestyle choices over the course of 16 weeks and 12 one hour classes.

Findings suggest that the HTT program is effective in improving nutrition, physical activity, social emotional knowledge and behaviors among child participants, as measured by self-reported pre- and post-test assessments. There were multiple statistically significant health behavior findings, indicating that the HTT program has an impact on fruit and vegetable consumption, eating as a family, increased water consumption, decreased soda consumption, positive self-talk, physical activity, and daily walking. Total knowledge increased by $22 \%$ on the 13 questions assessment. Each section of the assessment also saw significant knowledge increases from pre- to post-test: nutrition (28.2\%), social emotional (18.9\%), and physical activity (17.8\%).

HTT curriculum emphasizes that weight management is a family lifestyle change. Weight reduction does not happen instantly, and there are elements outside of nutrition and physical activity at play, such as readiness to change, social emotional factors, and self-concept. Many of the significant findings correspond to factors that parents are in control of: eating as a family, fruit and vegetable and soda or sugary drink consumption, planning and prepping food, and being physically active (whether by example or by encouraging family activities).

Aspects of the HTT curriculum are designed to help support parents as they make these changes for their family. All trained professionals are present at each HTT class, regardless of the focused topic that week. This means someone is available at all classes to answer any questions or give advice on anything that 
might have come up during the week. The program is also designed to taper towards the end; this gives families the chance to sustain lifestyle changes for longer intervals between classes while still offering support before the end of the program.

Improving knowledge, skills, and abilities amongst the child participants is important, but parents have much control over consumption and behaviors. In addition to targeting children, involving parents is essential for successful weight management programs. In a study looking at parental readiness to change in relation to child behaviors, parents that increased their stage of change on the TTM from pre-contemplation or contemplation to action or maintenance reported significant decreases in child fast food consumption and increases in child fruit and vegetable consumption [17]. Understanding both parent and child stage of change is important in the ongoing research for effective weight management strategies.

While nearly all of the results in this evaluation were positive, one item on the health behaviors assessment asked participants to report on the days per week they felt stressed. This result was statistically significant in the unfavorable direction. One explanation may be the timing of assessments. The pre-assessments correspond with the beginning of either the fall or spring school semester when students are unlikely to have tests and large amounts of homework. The post-assessments take place towards the end of the school semester when participants have more school work. Participants can also get better at self-reporting behaviors, which can distort the pre- to post-test change.

This evaluation indicates that an hourly, 16 week family based weight management program can successfully improve health knowledge, behaviors, and accelerate readiness to change to adopt healthier lifestyles. However, recent changes in the requirements for weight management programs have resulted in the HTT program to increase the dosage to 17 weeks. Due to this increase of dosage, the program will no longer taper at the end but will meet weekly. There are several limitations to the evaluation of this program. Body metrics are the only assessment that is not self-report, and social desirability and better awareness of behaviors at post-test may have influenced the results. There is also controversy in BMI as a metric. Child participants are growing and developing throughout the program, which makes a pre- post-assessment of BMI inaccurate. Baseline BMI does however provide parents and program facilitators with an initial indicator of their child's health. Finally, the Piers Harris assessment measures self-concept, but self-concept tends to not change as a result of a one-time intervention or isolated experience. Self-concept changes and develops over the course of a lifetime in response to environmental and developmental changes or as a result of changing priorities and values [17]. Other self-concept assessments that may be responsive to a one-time intervention are being explored.

Childhood obesity is a growing epidemic that must be addressed in a multi-disciplinary manner. Creating a relationship with a pediatric obesity clinic, such as the HSC can increase the total number of children and teens that are introduced to weight management programs like HTT. Participants in this study population significantly improved several body metrics, knowledge, health behaviors, and readiness to change assessments as a result of participating in a family based weight management program. Further follow-up with both child and parent participants is needed to measure the long-term impact of HTT.

\section{Acknowledgements}

HTT is funded through the St. Louis Children's Hospital Foundation (1001 Highlands Plaza Drive West, Suite 160 St. Louis, MO 63110). The curriculum was developed using CDC guidelines for physical activity and USDA Center for Nutrition Policy and Promotion guidelines for nutrition.

The HSC is on the Second Floor of St. Louis 

Obesity Intervention that Partners with an Obesity Clinic

Children's Hospital (1 Children's Place, St Louis, MO 63116). The staff include Janis Stoll, MD, Phillip Tarr, MD, Karen Jones, RN, Amy Lamb-Schnitzer, RN, Christina Stephan, RN, MSN, CPNP, Beeper Rauckman, RN, BSN, Jennifer Scholl, RN, MSN, CPNP, Janet Sokolich, RN, Sandy Summers, MSN, PNP-BC and Jill Schnurbusch, MS, CHES (HTT representative).

J. Sch. implemented the program. C. Cio. and M. Sch. conducted the literature search and data collection. C. Cio. and M Sch. completed the data analysis and interpretation. N. Koz, S. Rei and N. Gar provided critical review. All authors approved the final manuscript.

\section{Disclosure}

The authors declare no conflicts of interest.

\section{References}

[1] “Obesity Rates \& Trends Overview.” Retrieved December 16, 2016. http://stateofobesity.org/obesity-rates-trends-overview.

[2] "Nutrition and Weight Status.” Retrieved December 20, 2016.

https://www.healthypeople.gov/2020/topics-objectives/to pic/nutrition-and-weight-status/objectives.

[3] Centers for Disease Control and Prevention. 2015. “About Child \& Teen BMI.” Retireved December 20, 2016.

https://www.cdc.gov/healthyweight/assessing/bmi/childre ns_bmi/about_childrens_bmi.html.

[4] Nader, P. R., Obrien, M., Houts, R., Bradley, R., Belsky, J., Crosnoe, R., and Susman, E. J. 2006. “Identifying Risk for Obesity in Early Childhood.” Pediatrics 118 (3). doi:10.1542/peds.2005-2801.

[5] Freedman, D. S., Khan, L. K., Serdula, M. K., Dietz, W. H., Srinivasan, S. R., and Berenson, G. S. 2005. "The Relation of Childhood BMI to Adult Adiposity: The Bogalusa Heart Study.” Pediatrics 115 (1): 22-7. doi:10.1542/peds.2004-0220.

[6] Freedman, D. S., Dietz, W. H., Srinivasan, S. R., and Berenson, G. S. 2009. "Risk Factors and Adult Body Mass Index among Overweight Children: The Bogalusa Heart Study.” Pediatrics 123 (3): 750-7. doi:10.1542/peds.2008-1284.

[7] Daniels, S. R. 2009. "Complications of Obesity in
Children and Adolescents." International Journal of Obesity 33 (S1). doi:10.1038/ijo.2009.20.

[8] Whitlock, E. P. 2005. "Screening and Interventions for Childhood Overweight: A Summary of Evidence for the US Preventive Services Task Force.” Pediatrics 116 (1). doi:10.1542/peds.2005-0242.

[9] Morrison, K. M., Shin, S., Tarnopolsky, M., and Taylor, V. H. 2015. "Association of Depression \& Health Related Quality of Life with Body Composition in Children and Youth with Obesity.” Journal of Affective Disorders 172: 18-23. doi:10.1016/j.jad.2014.09.014.

[10] Kitzmann, K., Dalton, W., Stanley, C., Beech, B., Reeves, T., Buscemi, J., Egli, C., Gamble, H., and Midgett, E. 2010. "Supplemental Material for Lifestyle Interventions for Youth Who Are Overweight: A Meta-Analytic Review.” Health Psychology 19 (1): 91-101. doi:10.1037/a0017437.supp.

[11] Caprio, S. 2006. "Treating Child Obesity and Associated Medical Conditions.” The Future of Children 16 (1): 209-24. doi:10.1353/foc.2006.0002.

[12] Xanthopoulos, M. S., Moore, R. H., Wadden, T. A., Bishop-Gilyard, C. T., Gehrman, C. A., and Berkowitz, R. I. 2013. "The Association between Weight Loss in Caregivers and Adolescents in a Treatment Trial of Adolescents with Obesity." Journal of Pediatric Psychology 38 (7): 766-74. doi:10.1093/jpepsy/jst024.

[13] Kalarchian, M. A., Levine, M. D., Arslanian, S. A., Ewing, L. J., Houck, P. R., Cheng, Y., and Marcus, M. D. 2009. "Family-Based Treatment of Severe Pediatric Obesity: Randomized, Controlled Trial.” Pediatrics 124 (4): 1060-8. doi:10.1542/peds.2008-3727.

[14] Prochaska, J. O., and Velicer, W. F. 1997. "The Transtheoretical Model of Health Behavior Change.” American Journal of Health Promotion 12 (1): 38-48. doi:10.4278/0890-1171-12.1.38.

[15] Menezes, M. C. 2016. "Interventions Directed at Eating Habits and Physical Activity Using the Transtheoretical Model: A Systematic Review.” Nutrición Hospitalaria 33 (5). doi:10.20960/nh.586.

[16] Riebe, D., Greene, G. W., Ruggiero, L., Stillwell, K. M., Blissmer, B., Nigg, C. R., and Caldwell, M. 2003. "Evaluation of a Healthy-Lifestyle Approach to Weight Management.” Preventive Medicine 36 (1): 45-54. doi:10.1006/pmed.2002.1126.

[17] “Piers-Harris ${ }^{\mathrm{TM}}$ 2: Piers-Harris Children's Self-Concept.” https//www.bing,com/r?IG=3FAFB99739E04B9AB9D082F66885FA14 \&CID=11DD7BB7D1846A5D3CED7/F3D0796BB5\&nd=1\&h=M4-jf x8uPMVJ3hhepelyvmw09Lt0RAZQCvRpNyPo14\&v=1\&r=https//ww w.wpspublish.com/storep/2912/piers-hanis-2-piershamis-dildrens-self-conce pt-scale-secondedition\&p=DevEx.LB.1,5495.1. 